\title{
Randomised controlled trial of early prophylactic feeding vs standard care in patients with head and neck cancer
}

\author{
Teresa E Brown ${ }^{\star, 1,2}$, Merrilyn D Banks², Brett G M Hughes ${ }^{3,4}$, Charles Y Lin ${ }^{3,4}$, Lizbeth M Kenny ${ }^{3,4}$ \\ and Judith D Bauer ${ }^{1}$ \\ ${ }^{1}$ Centre for Dietetics Research (C-DIET-R), School of Human Movement and Nutrition Sciences, The University of Queensland, St \\ Lucia, Brisbane QLD 4072, Australia; ${ }^{2}$ Department of Nutrition and Dietetics, Royal Brisbane and Women's Hospital, Herston, \\ Brisbane QLD 4029, Australia; ${ }^{3}$ Cancer Care Services, Royal Brisbane and Women's Hospital, Herston, Brisbane QLD 4029, \\ Australia and ${ }^{4}$ Faculty of Medicine, The University of Queensland, St Lucia, Brisbane QLD 4072, Australia
}

Background: Weight loss remains significant in patients with head and neck cancer, despite prophylactic gastrostomy and intensive dietary counseling. The aim of this study was to improve outcomes utilising an early nutrition intervention.

\begin{abstract}
Methods: Patients with head and neck cancer at a tertiary hospital in Australia referred for prophylactic gastrostomy prior to curative intent treatment were eligible for this single centre randomised controlled trial. Exclusions included severe malnutrition or dysphagia. Patients were assigned following computer-generated randomisation sequence with allocation concealment to either intervention or standard care. The intervention group commenced supplementary tube feeding immediately following tube placement. Primary outcome measure was percentage weight loss at three months post treatment.
\end{abstract}

Results: Recruitment completed June 2015 with 70 patients randomised to standard care (66 complete cases) and 61 to intervention (56 complete cases). Following intention-to-treat analysis, linear regression found no effect of the intervention on weight loss $(10.9 \pm 6.6 \%$ standard care vs $10.8 \pm 5.6 \%$ intervention, $P=0.930)$ and this remained non-significant on multivariable analysis $(P=0.624)$. No other differences were found for quality of life or clinical outcomes. No serious adverse events were reported.

Conclusions: The early intervention did not improve outcomes, but poor adherence to nutrition recommendations impacted on potential outcomes.

Treatments for head and neck cancer (HNSCC), including surgery, radiotherapy and chemotherapy have a number of side effects, which can impact on nutritional intake (Talwar and Findlay, 2012), with multi-modality therapy having higher toxicity (Moroney et al, 2017). As a consequence, many patients experience significant weight loss and develop malnutrition during the course of treatment (Hebuterne et al, 2014). This is associated with a number of detrimental outcomes, including reduced physical functioning, quality of life and immune function, and increased treatment interruptions, toxicities, hospital admissions, and mortality (Gorenc et al, 2015). Critical weight loss of 5\% or more during treatment has been associated with worse survival outcomes (Langius et al, 2013a), demonstrating the importance of optimal nutrition to minimise weight loss. Improvements in nutritional status have also been linked to improved aspects of quality of life (Isenring et al, 2004; Ravasco et al, 2004).

A number of systematic reviews have recommended the importance of dietary counselling to improve nutritional and

*Correspondence: TE Brown; E-mail: Teresa.brown@uqconnect.edu.au

Received 13 December 2016; revised 21 April 2017; accepted 24 April 2017; published online 23 May 2017

(C) 2017 Cancer Research UK. All rights reserved 0007-0920/17 
patient outcomes (Garg et al, 2010; Langius et al, 2013b); however, the role of tube feeding remains unclear due to a lack of welldesigned clinical trials to inform optimal tube type and timing of placement (Orphanidou et al, 2011; Nugent et al, 2013; Wang et al, 2014). A recent meta-analyses comparing outcomes with nasogastric tubes and either prophylactic or reactive gastrostomy tubes, found the prophylactic tubes resulted in a reduction in treatment interruptions and nutrition related hospital admissions, but had no superiority in managing nutritional status (Zhang et al, 2016). Either way, clinically significant weight loss of $>10 \%$ occurs with either the prophylactic or reactive approach (Chang et al, 2009; Clavel et al, 2011; Rutter et al, 2011; Silander et al, 2012; Olson et al, 2013).

Of those studies that report specific details on the actual timing of the commencement of nutrition support through the prophylactic gastrostomy, the majority are after the commencement of treatment when it becomes clinically indicated in response to deterioration in swallowing or nutritional status (Scolapio et al, 2001; Rayker et al, 2009; Nugent et al, 2010). Hence, it is not surprising that there are similar nutrition outcomes with reactive tube use; as although the prophylactic tube is in situ and ready for access, the initiation and indication for nutrition support is reactive in both groups. Some studies have reported the commencement of enteral feeds prior to treatment (Marcy et al, 2000; Beer et al, 2005; Nguyen et al, 2006; Wiggenraad et al, 2007) but these patients had poor nutritional status or dysphagia at baseline, and therefore nutrition support was clinically indicated immediately.

This is the first study to our knowledge to trial an early prophylactic tube feeding intervention prior to treatment in a target population who are primarily well-nourished with minimal dysphagia. This group may be considered to have low motivation to use their tube as there is no current obvious problem with eating, but despite good baseline nutritional and swallowing status, these patient have still been shown to have high rates of tube feeding and weight loss during treatment (Brown et al, 2015). The validation of the protocol used at our institute to identify patients for prophylactic gastrostomy also supports this finding (Brown et al, 2016). The criteria used in this protocol are primarily based on tumour site and treatment, and so the majority of patients selected are those receiving chemoradiotherapy for unresectable tumours or as organ-preserving treatment. By increasing nutritional intake through prophylactic supplementary tube feeding, it was assumed this would assist in reducing weight loss. The additional rational for this interventional approach was based on the psychological impact of gastrostomy tube placement, including anxiety and fear associated with tube use (Salas et al, 2009; Merrick and Farrell, 2012). Therefore, this period of early tube feeding could assist patients to adapt to the tube more easily.

The aim of this study is to compare nutritional, clinical and patient outcomes following an early tube feeding approach via the prophylactic gastrostomy $v s$ standard care of commencing tube feeding via the prophylactic gastrostomy when clinically indicated. The null hypothesis being no difference in the outcomes between the two groups. It is anticipated that the intervention group will have improved nutritional outcomes, according to an established nutrition framework, which in turn is expected to improve patient outcomes, such as quality of life, and other clinical outcomes (Splett, 1996).

\section{MATERIALS AND METHODS}

This was a single-centre randomised controlled trial conducted in Queensland, Australia. The full protocol is published (Brown et al, 2014). The study design was a parallel group study using equal randomisation $(1: 1)$. No changes occurred to the study design or outcome measures following commencement.

Participants and study setting. Patients with HNSCC were eligible for the study if referred for a prophylactic gastrostomy prior to treatment. A local protocol was used by the multidisciplinary clinic to identify high nutrition risk patients suitable for prophylactic gastrostomy (Brown et al, 2016); however, the final decision of tube placement was made by the treating team. Excluded patients included: age $<18$, pregnant, cognitively impaired or with an intellectual disability or mental illness, planned for non-curative intent treatment, or if diagnosed as severely or moderately malnourished with significant dysphagia requiring a liquid or puree texture modified diet.

The study was approved by the Royal Brisbane and Women's Hospital Human Research Ethics Committee on 19 July 2012 (HREC/12/QRBW/162) and The University of Queensland Medical Research Ethics Committee on 8 August 2012 (2012000890). All patients provided written informed consent to participate. This trial has been registered in the Australian New Zealand Clinical Trials registry as ACTRN12612000579897. Available at http://www.anzctr.org.au.

Patients were recruited from the Royal Brisbane and Women's Hospital from September 2012 to June 2015. This is a major tertiary/quaternary hospital providing specialist cancer care services to patients throughout Queensland, Australia. All patients see the dietitian and speech pathologist as part of routine care, with access to other allied health services as required. Dependent on the patient's home address and local health service district location, follow-up care post treatment may continue at the tertiary centre or be transferred to a regional cancer centre. Level of allied health services and thus access to follow-up care at each regional centre may vary, particularly for patients from rural or remote areas.

Interventions. All patients received education on the care of their feeding tube during their overnight elective admission for gastrostomy placement. Prior to discharge patients were randomly allocated to either the standard care or intervention arm and booked into the joint dietitian and speech pathology clinic for weekly review during treatment.

In the standard care arm, patients were commenced on enteral nutrition via their prophylactic gastrostomy by the dietitian when indicated. Indicators included: oral intake $<60 \%$ of estimated energy requirements (based on $125-145 \mathrm{~kJ} \mathrm{~kg}^{-1}$ ) for a period of, or anticipated to be, >10 days; the patient was unable to maintain weight; the patient required significant texture modification of diet; or the patient had increased or uncontrolled nutrition impact symptoms. The regimen was determined by the clinical dietitian to suit the patients' individual requirements and adapted as required during treatment.

For patients in the intervention group, this meant initiation of enteral nutrition via their prophylactic gastrostomy immediately following tube placement prior to commencement of treatment. The prophylactic enteral nutrition was in addition to their current oral intake and consisted of $2 \times 200 \mathrm{ml}$ bolus feeds $\left(1.5 \mathrm{kcal} \mathrm{ml}^{-1}\right.$ polymeric formula with fibre) per day. Patients were provided with weekly supplies and were given guidance and suggestions on how to incorporate the enteral feeding into their daily routine (e.g., behavioural strategies and reminders; appropriate timings for administration, such as between meals). This enteral nutrition recommendation was continued until completion of treatment, and was increased as necessary during treatment if the patient had any of the clinical indicators for starting enteral nutrition as described in standard care. If patients required an increase in enteral nutrition, they were converted to a home enteral nutrition prescription and were required to pay a co-payment for the product as per standard care. If patients described enteral feed intolerance, an alternative non-fibre formula was trialled. 
To monitor adherence, patients in both arms were asked to maintain a self-reporting diary of their daily prescribed enteral nutrition intake, and any barriers to this prescription. These were verified by the clinical dietitian on weekly review. Overall adherence was defined as achieving $\geqslant 75 \%$ of prescribed enteral nutrition intake (Hubbard et al, 2012). All patients were encouraged to maintain oral intake as much as possible during treatment and as long as it remained safe to do so as per the speech pathologist. On completion of treatment, all patients were referred to their local dietitian service for ongoing care as required.

Outcomes. The primary endpoint was percentage weight change with additional nutrition outcomes, including body composition (fat mass and fat-free mass) and nutritional status. Weight and body fat percentage were measured on digital bioelectrical impedance analysis (BIA) scales (Tanita body composition monitor BC-582 (Tanita Corporation, Japan)) at recruitment (baseline) and three months post treatment. Patients were asked to remove shoes, socks and outer clothing, and to empty their pockets. Nutritional status was assessed by the Patient-Generated Subjective Global Assessment tool (PGSGA) (Bauer et al, 2002), a validated tool recommended to assess nutritional status in patients with cancer (Isenring et al, 2013).

The secondary endpoint was quality of life, which was assessed using the European Organisation for Research and Treatment of Cancer (EORTC) tools (Aaronson et al, 1993). The questionnaires included the quality of life of cancer patients (EORTC QLQ-C30) and the module for head and neck (EORTC QLQ-H\&N35). The raw data was transformed into scores ranging from 0-100, following established procedures (Fayers et al, 2001). A higher score for global quality of life and functioning scales was indicative of higher quality of life and function. A higher score for symptom scales was indicative of a higher level of symptom burden. Guidelines for interpretation of longitudinal changes in quality of life scores for EORTC QLQ-C30 were used to determine clinical impact (Cocks et al, 2012) and these were graded as either an improvement or deterioration, with trivial, small, medium or large clinical effect.

Tertiary endpoints included tolerance to chemotherapy and radiotherapy, rate of unplanned hospital admissions and gastrostomy complications. Dose of chemotherapy and radiotherapy received were recorded. Delays to radiotherapy were recorded as days beyond expected finish date and a prolonged treatment time was defined as those $>7$ days. Completion of planned chemotherapy was defined as either completion of three cycles of high-dose cisplatin, seven cycles of weekly cisplatin or eight cycles of cetuximab. Completion of target dose was defined as $\geqslant 200 \mathrm{mg} \mathrm{m}^{-2}$ cisplatin or $\geqslant$ six cycles of cetuximab. Reasons for changes to chemotherapy were coded as toxicities (gastrointestinal, haematological, renal, or other). Unplanned hospital admissions and length of stay were prospectively recorded during treatment and classified as either a medical admission, or management of gastrostomy complications or management of nutrition impact symptoms. Gastrostomy complications were recorded during the first three months post insertion. Major complications were defined as those requiring hospital admission. Minor complications were defined as site infections or other stoma issues such as hypergranulation, excoriation, erythema, pressure injury or leakage.

Monthly follow-up was completed via the telephone to assess gastrostomy use and date of removal for up to 6 months post treatment. If the tube was still in situ at 6 months, then follow-up was repeated at 12 months. The day of gastrostomy removal was recorded in relation to the day of treatment completion.

Treatment response was assessed via the 3-month post treatment PET/CT scan and defined as either complete metabolic response, or persistent disease requiring salvage surgery or persistent disease requiring palliative care. Survival outcomes were assessed at 12 months post treatment and time (in months) to disease relapse and/or death were recorded.

To enhance the quality of the measurements and to reduce any inter-rater variability in assessment, the data was collected by the designated research dietitian. If patients failed to attend their follow-up appointment, quality of life data were obtained by telephone interview and/or completion of postal surveys, and weight and the PGSGA data were obtained following face-to-face assessment by the local dietitian.

Sample size. The primary outcome for the study was a continuous response variable of percentage weight loss. The aim was to reduce the absolute amount of percentage weight loss by $5 \%$ in the intervention group compared to the percentage weight loss seen in the standard care group. A sample size of 123 patients was required to detect a $5 \%$ difference in percentage weight loss between the intervention and control groups, with a two-sided 5\% significance level, power of $80 \%$ and an attrition factor of $10 \%$. Recruitment continued until sample size was attained.

Randomisation. Patients were stratified according to baseline nutritional status (well nourished (PGSGA A) vs moderate/ suspected malnutrition (PGSGA B)), and then randomly assigned to one of the two groups (with an allocation ratio $1: 1$ ) following simple randomisation procedures. The randomisation sequence was computer-generated using MS Excel. This allocation sequence was concealed to the researcher enrolling participants, but the participants, healthcare team or outcome assessor were not able to be blinded to the allocation.

Statistical methods. Baseline participant characteristics were summarised using mean and s.d. for continuous variables and frequency, and percentage for categorical variables. Differences between groups were assessed using $\chi^{2}$ tests for categorical variables (or Fishers exact test if assumptions could not be met due to small expected cell counts) and two sample $t$-tests for continuous variables (or two-sample Wilcoxon tests, if assumptions could not be met for normally distributed data and homogeneity of variance). Normal distribution was formally assessed using Shapiro-Wilk test and variance assessed using Levene's test.

Analysis was performed on an intention-to-treat basis. To determine the effect of the nutrition intervention group $v s$ the control group (the independent categorical variable) on the primary outcome of \% weight loss (the dependent continuous variable), analysis of variance (ANOVA) was used. For the secondary outcomes (\% fat-free mass, \% fat mass, PGSGA score and quality of life scores), the mean change over time from baseline to 3 months was also analysed between groups using ANOVA. Bivariate analysis determined the association of any other independent variables on the outcome measures. Any variables with statistically significant associations $(P<0.1)$ were entered into a linear regression model (or logistic regression for any binary outcome variables). Any variables with baseline differences between groups were also added into the model as covariates to adjust for any confounding effects. Final variable selection for each multivariable model used Akaike's Information Criteria (AIC) with a backward stepwise algorithm. The final models reported were those with the smallest objective AIC. Time to event outcomes (gastrostomy removal, disease relapse, death) were analysed using the Kaplan-Meier method with the log rank test.

Statistical significance was set at $P<0.05$. The data were analysed using R Commander Version 2.1-7 and R version 3.1.3 (2015-03-09) (R Foundation for Statistical Computing, Vienna, Austria). For any results that were not statistically significant, any relevant clinically significant results were reported. 


\section{RESULTS}

Patient characteristics. Eligible patients $(n=174)$ were invited to participate in the study between September 2012 to June 2015 with 131 patients recruited and randomised (Figure 1). The final 12 month outcomes were completed in August 2016 and all patients were analysed on an intention-to-treat basis. Patient, clinical and treatment characteristics and their baseline nutrition measures are summarised in Table 1 and baseline quality of life measures are available in Supplementary Table 1. In the standard care group, two patients received surgery alone and one patient had radiotherapy alone. There were five patients in the intervention group who had post-operative radiotherapy. All other patients in the study received definitive or adjuvant chemoradiotherapy (94\%), of which five also had neoadjuvant chemotherapy. Radiotherapy was delivered by helical intensity modulated radiotherapy for $98 \%$ of patients, with definitive doses at $>66 \mathrm{~Gy}(n=111)$ and adjuvant doses at $60-66 \mathrm{~Gy}(n=12)$. Most patients had their gastrostomy placed via endoscopy (93\%) vs radiologically (7\%), a median of 5 days prior to treatment in each group $(P=0.277)$.

Nutrition outcomes. There were no significant differences for weight loss, body composition or nutritional status once adjusted for any differences at baseline or any other confounding variables in multivariable models (Table 2). The only predictors of weight loss in the multivariate model were baseline BMI and P16 status; with every 10 unit increase in BMI, there was $\sim 4 \%$ more weight loss, and patients with p16 negative disease had $\sim 4 \%$ less weight loss than those with p16 positive disease. No variables were identified to predict loss of fat-free mass. Predictors of other nutritional outcomes (fat mass and PGSGA) from multivariable models are summarised (Supplementary Table 2).

Quality of life outcomes. There were no statistical differences in any of the domains of quality of life or functional scales after adjusting for any baseline differences (Supplementary Table 3). Multivariable models were created for global quality of life, functioning scales and selected symptoms scales for which the intervention may have impacted on (e.g., fatigue, appetite, gastrointestinal symptoms, dysphagia and social eating); however, no significant effects of the intervention were found following adjustment for other confounding variables (Supplementary Table 4). On review of the clinical impact of quality of life (EORTC QLQ-C30), the majority of domains showed some degree of deterioration over time (particularly loss of appetite, role functioning and fatigue) but comparisons between groups was limited due to baseline differences. The only exception was social functioning, which was similar at baseline, but clinical differences

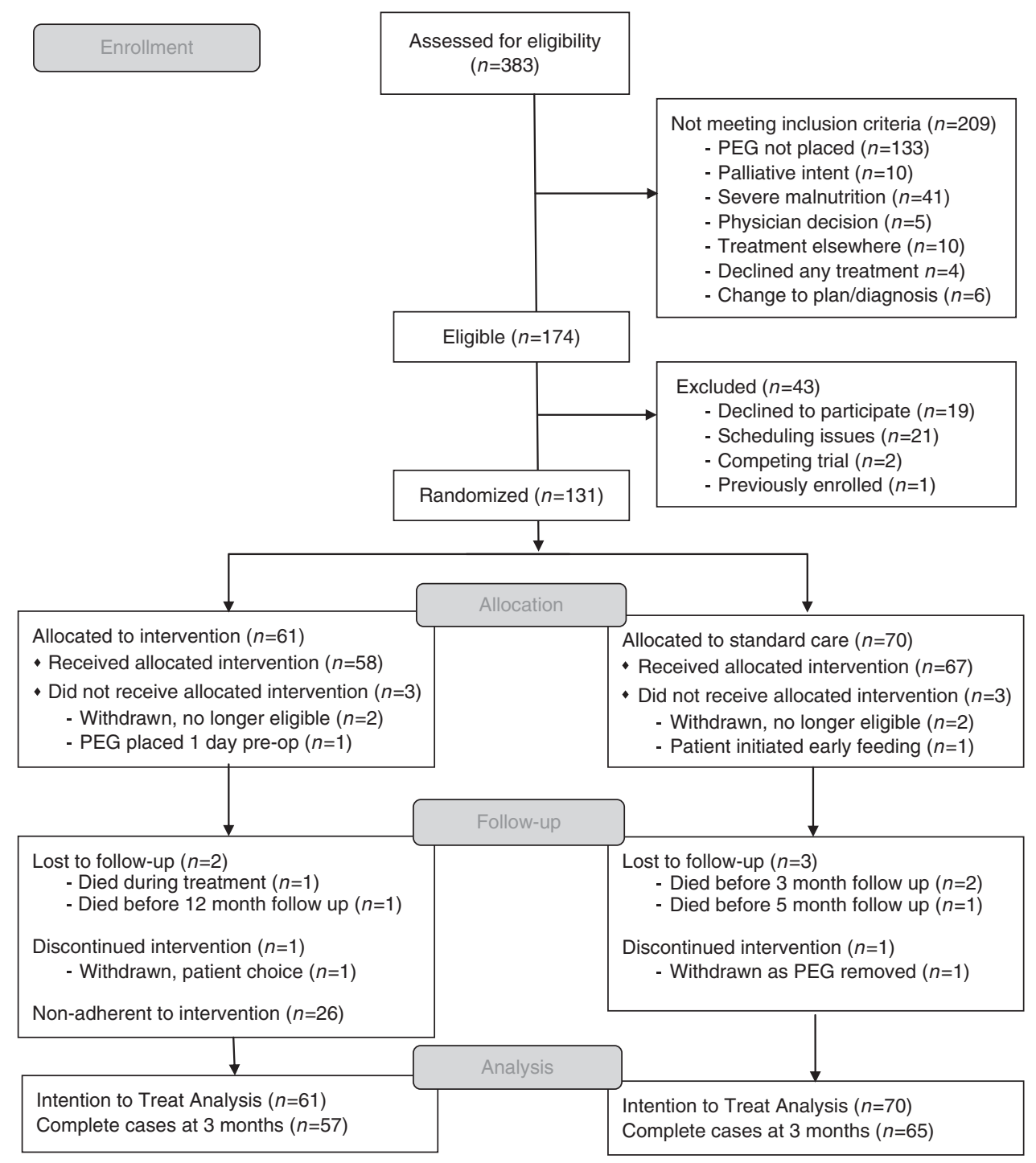

Figure 1. CONSORT flow diagram. Abbreviation: PEG = gastrostomy. 
Table 1. Summary of patients enrolled in the trial and comparison of baseline characteristics between standard care and intervention groups

\begin{tabular}{|c|c|c|c|c|}
\hline Variable & Total $(n=131)$ & Standard care $(n=70)$ & Intervention $(n=61)$ & $P$-value \\
\hline \multicolumn{5}{|l|}{ Age } \\
\hline Years (mean \pm s.d.) & $60.5 \pm 10.1$ & $60.0 \pm 10.9$ & $61.1 \pm 9.1$ & 0.558 \\
\hline $\begin{array}{l}\text { Male } \\
\text { Female }\end{array}$ & $\begin{array}{r}115(88) \\
16(12)\end{array}$ & $\begin{array}{l}59(84) \\
11(16)\end{array}$ & $\begin{array}{c}56(92) \\
5(8)\end{array}$ & 0.19 \\
\hline \multicolumn{5}{|l|}{ Smoking history } \\
\hline Current & $24(18)$ & $17(24)$ & $7(11)$ & 0.147 \\
\hline \multicolumn{5}{|l|}{ Tumour site } \\
\hline Oral cavity & $14(11)$ & $7(10)$ & $7(11)$ & \multirow[t]{4}{*}{0.885} \\
\hline Oropharynx & $101(77)$ & $56(80)$ & 45 (74) & \\
\hline Nasopharynx & $4(3)$ & $2(3)$ & $2(3)$ & \\
\hline Hypopharynx & $8(6)$ & $3(4)$ & $5(8)$ & \\
\hline TO & $1(1)$ & $0(0)$ & $1(2)$ & \multirow[t]{5}{*}{0.111} \\
\hline $\mathrm{T} 1$ & $8(6)$ & $4(5)$ & $4(7)$ & \\
\hline T2 & $44(34)$ & $20(29)$ & 24 (39) & \\
\hline T3 & $37(28)$ & $26(37)$ & 11 (18) & \\
\hline T4 & $41(31)$ & $20(29)$ & $21(34)$ & \\
\hline \multicolumn{5}{|l|}{$\mathrm{N}$ stage } \\
\hline NO & $12(9)$ & $7(10)$ & $5(8)$ & \multirow{6}{*}{0.629} \\
\hline N1 & $13(10)$ & $8(11)$ & $5(8)$ & \\
\hline N2a & $4(3)$ & $1(1)$ & $3(5)$ & \\
\hline $\mathrm{N} 2 \mathrm{~b}$ & $55(42)$ & $30(43)$ & $25(41)$ & \\
\hline N2c & $42(32)$ & $20(29)$ & $22(36)$ & \\
\hline N3 & $5(4)$ & $4(6)$ & $1(2)$ & \\
\hline \multicolumn{5}{|l|}{ P16 status } \\
\hline Weekly cisplatin & $25(21)$ & $12(19)$ & $13(25)$ & \multirow{2}{*}{0.382} \\
\hline Cetuximab & $24(21)$ & $16(25)$ & $8(15)$ & \\
\hline Weight loss 6 months & & & & \\
\hline $\mathrm{Nil}$ & $51(39)$ & $22(31)$ & $29(48)$ & 0.266 \\
\hline$<5 \%$ & $43(33)$ & 27 (39) & $16(26)$ & \\
\hline $5-10 \%$ & $22(17)$ & $12(17)$ & $10(16)$ & \\
\hline$\geqslant 10 \%$ & $15(11)$ & $9(13)$ & $6(10)$ & \\
\hline Nutritional status & & & & \\
\hline PGSGA A & $99(76)$ & $50(71)$ & $49(80)$ & 0.237 \\
\hline PGSGA B & $32(24)$ & $20(29)$ & $12(20)$ & \\
\hline PGSGA C & $0(0)$ & $0(0)$ & $0(0)$ & \\
\hline Diet & & & & \\
\hline Full & $87(66)$ & $47(67)$ & $40(66)$ & 0.579 \\
\hline Soft & 35 (27) & $20(29)$ & $15(25)$ & \\
\hline Minced & $8(6)$ & $3(4)$ & 5 (8) & \\
\hline Puree & $1(1)$ & $0(0)$ & $1(1)$ & \\
\hline Anthropometry & & & & \\
\hline Weight (kg) & $82.6 \pm 18.7$ & $79.7 \pm 18.7$ & $85.8 \pm 18.4$ & 0.063 \\
\hline Fat-free mass $(\mathrm{kg})$ & $59.2 \pm 10.4$ & $57.6 \pm 10.5$ & $60.9 \pm 10.1$ & 0.073 \\
\hline BMI $\left(\mathrm{kg} \mathrm{m}^{-2}\right)$ & $27.2 \pm 5.5$ & $26.4 \pm 5.7$ & $28.1 \pm 5.2$ & 0.078 \\
\hline Nutrition risk & & & & \\
\hline PGSGA score (median, range) & $6(1-21)$ & $6(1-21)$ & $5(1-20)$ & 0.052 \\
\hline
\end{tabular}




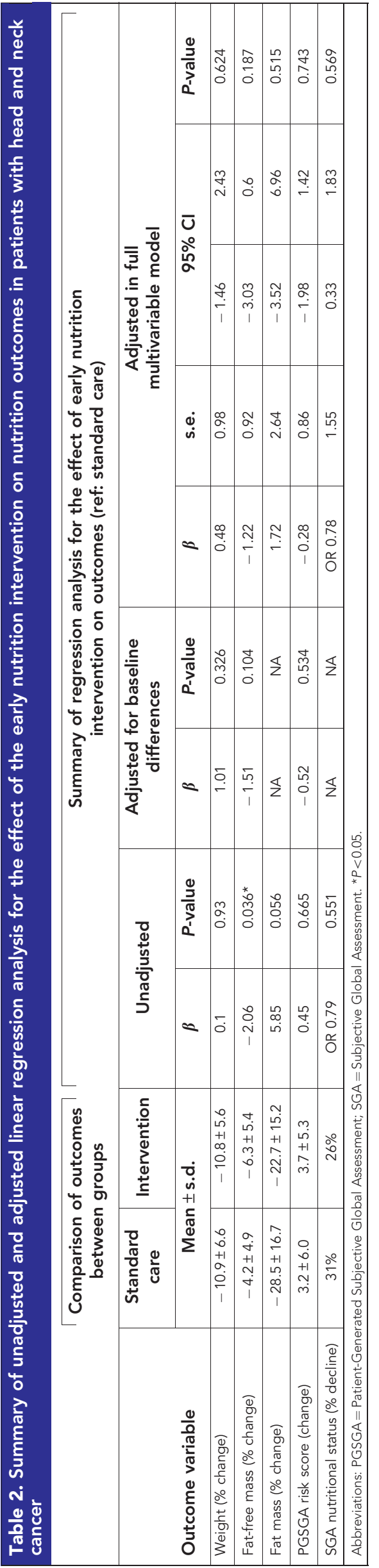

were seen with a medium deterioration in the intervention group and no change in the standard group (Supplementary Table 5).

Radiotherapy tolerance. The proportion of patients completing planned radiotherapy treatment was $100 \%$ in standard care $(n=65$ of 65$)$ and $95 \%$ in the intervention group ( $n=55$ of 58$)$ $(P=0.102)$. Two patients in the intervention failed to attend scheduled treatment (one patient completed 62 of 70 Gy and one patient completed 66 of $70 \mathrm{~Gy}$ ), and one further patient died during treatment from liver failure due to complications of chemotherapy. There was no difference in rates of treatment replanning (22\% standard care $v s 19 \%$ intervention, $P=0.723)$ or delays to radiotherapy treatment $(n=9$ standard care $v s \quad n=7$ intervention, $P=0.381$ ). Three patients in the intervention and one patient in standard care had a prolonged treatment time, which were due to: admission for myocardial infarction; admission to a regional hospital for neutropenia and infection; admission to manage severe mucositis and secretions; and identification of a new node requiring re-scanning and planning.

Chemotherapy tolerance. Although only approximately half of patients completed their planned chemotherapy prescription $(59 \%$ standard care $v s 51 \%$ intervention, $P=0.361$ ), almost all completed the target dose of chemotherapy (95\% standard care vs $98 \%$ intervention, $P=0.407)$. Overall, there was no statistical difference in the changes to the planned chemotherapy (45\% standard care $v s$ $53 \%$ intervention, $P=0.418)$. The toxicity reasons for deviations from the prescribed chemotherapy plan were similar in each group: haematological (42\%), renal (27\%), gastrointestinal (5\%) and other (22\%).

Unplanned admissions. Unplanned admissions affected approximately half of all patients in each group (47\% standard care vs $57 \%$ intervention, $P=0.270$ ) with no statistical differences for reasons for admissions or associated length of stay (Table 3). Nutritionrelated admissions accounted for $47 \%$ of all admission events in the standard care group ( $n=20$ out of 43$)$ and $53 \%$ in the intervention group ( $n=20$ out of 38 ).

Gastrostomy outcomes. There was no gastrostomy placementassociated mortality. Major tube complications were similar in each group ( $12 \%$ standard care $v s 10 \%$ intervention, $P=0.775$ ). Pain management accounted for $45 \%$ of all major complications ( $n=9$ out of 20). Minor tube complications affected $40 \%$ of patients in both groups $(P=0.995)$, and events included: site infections requiring oral antibiotics $(n=27)$, other site/stoma complications $(n=29)$. Fifty percent of all patients had commenced clinically indicated tube feeding by week three. Tube use was high at the end of treatment with no differences between groups ( $88 \%$ standard care and $86 \%$ intervention, $P=0.776)$. The mean day of tube removal from completion of treatment was $104.7 \pm 54.0$ days in the standard care group $v s 115.5 \pm 62.5$ days in the intervention group $(P=0.333)$.

Survival and disease outcomes. The outcomes on PET at 3 months post treatment were no different between groups $(P=0.661)$. A complete response was seen in $78 \%$ of patients in standard care $(n=49)$ and $81 \%$ in the intervention $(n=42)$. Salvage surgery for persistent disease was carried out for six patients in each group, and palliative care was required for eight patients in standard care and four patients in the intervention. At 12 months, there were no differences in disease-free survival or overall survival, with 19 cases of disease relapse in the standard care group and 10 cases in the intervention group $(P=0.135)$ (Figure 2A) and six and five deaths in the standard care and intervention group, respectively $(P=0.946)$ (Figure $2 \mathrm{~B}$ ).

Additional analysis. Adherence to the intervention was defined as consuming $\geqslant 75 \%$ of the prescribed enteral feeding and unfortunately this was only achieved by $51 \%$ of patients. Due to this poor 
Table 3. Summary of the effect of the early nutrition intervention on unplanned admissions and LOS in patients with head and neck cancer

\begin{tabular}{|c|c|c|c|}
\hline & Standard care & Intervention & $P$-value \\
\hline Total number of patients with an unplanned admission & 31 & 33 & 0.27 \\
\hline $\begin{array}{l}\text { Reason for unplanned admission } \\
\text { Clinical } \\
\text { Nutrition } \\
\text { G tube }\end{array}$ & $\begin{array}{r}15(48) \\
20(65) \\
8(26)\end{array}$ & $\begin{array}{r}12(36) \\
20(61) \\
6(18)\end{array}$ & $\begin{array}{l}0.33 \\
0.747 \\
0.461\end{array}$ \\
\hline $\begin{array}{l}\text { LOS per unplanned admission } \\
\text { Clinical } \\
\text { Nutrition } \\
\text { G tube }\end{array}$ & $\begin{array}{l}3(1-23) \\
4(1-24) \\
3(1-22)\end{array}$ & $\begin{array}{l}6(1-36) \\
5(2-25) \\
1(1-2)\end{array}$ & $\begin{array}{l}0.182 \\
0.981 \\
0.154\end{array}$ \\
\hline
\end{tabular}

adherence, a per-protocol analysis was also performed to compare those patients who adhered to the intervention $(n=29)$ to standard care $(n=57)$, but no significant differences were found for any outcomes.

When adherence to the therapeutic phase of feeding was compared (i.e., when tube feeding became clinically indicated in each group) the intervention group had a higher adherence (58 vs $38 \%, P=0.037)$. Adherent patients $(n=49)$ had less weight loss than non-adherent patients $(n=55) \quad(-10.3$ vs $-12.6 \%$, $P=0.038)$.

Weight outcomes at the end of treatment were reviewed using routine clinical data. Weight change was $-6.7 \pm 5.3 \%$ in standard care $v s-6.1 \pm 4.5 \%$ in the intervention group on an intention-totreat basis $(P=0.466)$ and $-5.6 \pm 4.6 \%$ in the adherent intervention group on a per-protocol basis $(P=0.299)$.

\section{DISCUSSION}

This randomised controlled trial investigating an early tube feeding intervention was primarily conducted in a well-nourished group of patients with HNSCC who had a prophylactic gastrostomy placed prior to treatment without any significant dysphagia. The results from the intention-to-treat analysis found no impact on weight loss outcomes at three months post treatment, with both groups losing $\sim 10 \%$ of their body weight. Consequently, there were no statistical differences in any of the other secondary outcomes in regards to nutritional status, body composition, quality of life or other clinical outcomes.

The overall body composition results are very similar to a recent report (although they measured outcomes at one month post treatment), with an absolute percentage fat mass loss of $3.9 \%$ and a FFM loss of $2.4 \mathrm{~kg}$, vs our measures of $4.3 \%$ and $3.3 \mathrm{~kg}$ (De Carvalho et al, 2015). One study has reported lean mass accounts for $62 \%$ of weight loss mass (Jager-Wittenaar et al, 2011); however, this present study found the reverse with fat mass accounting for $67 \%$ of weight loss. The preservation of fat-free mass with nutrition intervention has been reported in other studies (Isenring et al, 2003). Further research is required on changes in body composition during and post treatment to understand any long term implications. Meanwhile, routine monitoring of body composition is suggested as this provides more information than body weight alone.

This is the first study to report on outcomes following an early prophylactic tube feeding intervention. There has been one other study, which has described an early nutrition intervention of dietary counseling pre-chemoradiotherapy compared to a historical control group, which found statistically significantly less weight loss, fewer breaks in radiotherapy and less unplanned admissions (Paccagnella et al, 2010). However, their early nutrition intervention was essentially equivalent to our control group. A similar style of study has recently been undertaken in patients
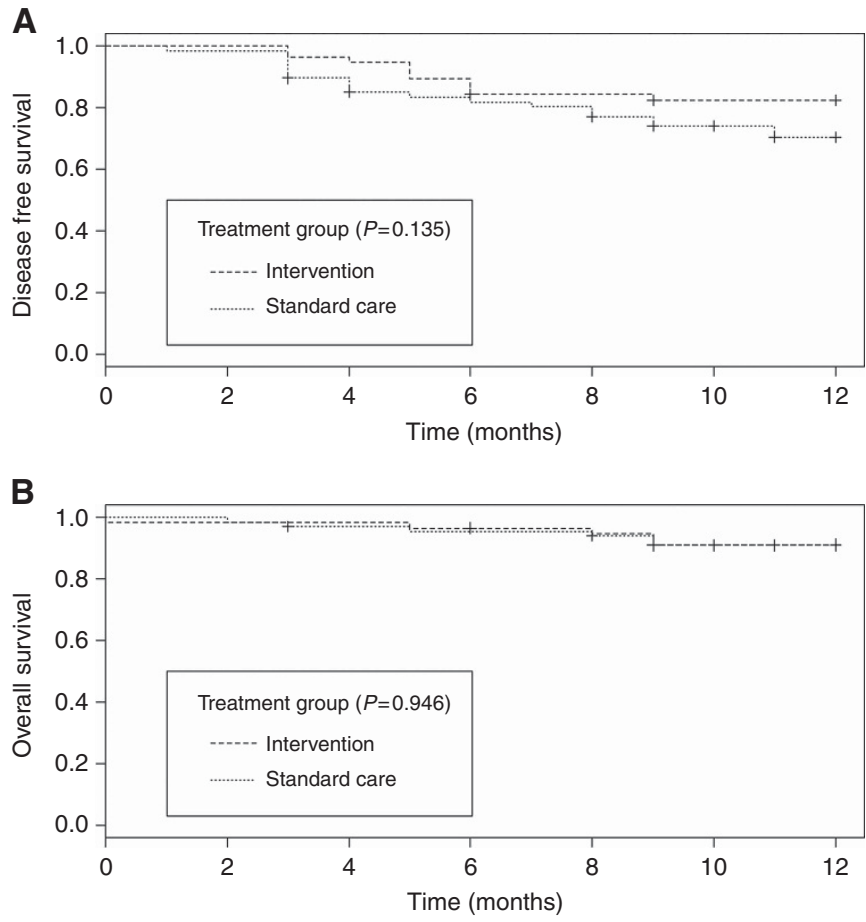

Figure 2. The effect of the early nutrition intervention on survival outcomes in patients with head and neck cancer. Kaplan-Meier curves to compare disease-free survival (A) and overall survival (B) at twelve months post-treatment between standard care and intervention groups.

with lung cancer (Kiss et al, 2016). Our current study design initiates supplementary prophylactic tube feeding prior to commencement of treatment and so could be described as an even more intensive early intervention.

The only other study to our knowledge, which has looked at early nutrition interventions in patients with HNSCC, is a secondary analysis in patients undergoing definitive radiotherapy as part of a larger prospective RCT $(n=1073)$ (Rabinovitch et al, 2006). Their results showed that although patients who received nutrition support prior to treatment had benefits in terms of less weight loss and less grade three or four mucositis, they ultimately had poorer overall survival (Rabinovitch et al, 2006). Their patients who received pretreatment nutrition support had more advanced stage tumours, lower performance status, more weight loss in last 6 months and more grade three and four dysphagia at baseline, and the detrimental impact of malnutrition at diagnosis on overall survival is well known (Datema et al, 2011). The methodological limitations of these exploratory findings are acknowledged by the authors and they recommend that RCT's are required to explore how nutrition 
support in the malnourished patient may negatively impact upon treatment outcomes (Rabinovitch et al, 2007); however, there have been no further studies in this field. Other factors also need to be taken into consideration in interpretation of these findings such as evolving treatment and disease, with patients now primarily receiving chemoradiotherapy (compared to radiotherapy alone) and a higher incidence of human papilloma virus (HPV) associated tumours (Hocking et al, 2011). The profile of patients selected for our study was also quite different as the eligibility criteria focused on well-nourished patients with minimal dysphagia. Given critical weight loss during treatment is also an independent prognostic indicator of worse disease-specific and overall survival rates (Langius et al, 2013a) and even subtle weight loss of $2.4 \%$ is related to decreased survival (Martin et al, 2015), these studies highlight the importance of including mortality as an outcome for any nutrition intervention studies. Our 1-year survival outcomes to date have not demonstrated any differences with respect to the timing of nutrition intervention, although this was not the primary outcome and sample size may be a limiting factor to draw any firm conclusions. Further survival analysis is planned at 5 years post treatment.

One of the potential reasons for the negative findings from this trial was the poor adherence to the prescribed nutrition intervention (51\%), which was much lower than expected. A study in hospitalised patients found $87 \%$ of prescribed tube feedings were met, however this was in a group of patients that were entirely dependent on enteral nutrition (Van den Broek et al, 2009). A systematic review on adherence to prescribed oral nutrition supplements, which is perhaps more akin to the supplementary bolus feeds used in this study, found a mean adherence of $79 \%$ (Hubbard et al, 2012). The prescribed daily tube feeds for the intervention were provided to the patients at no extra cost to facilitate adherence, as it has been shown that provision of complimentary nutritional supplements can improve outcomes (Lee et al, 2008), but this did not appear to help in this case. Key barriers patients encountered to tube feeding recommendations were nausea and early satiety, as well as a perception that the extra tube feeding was not required as patients felt they were eating normally. Further qualitative research is recommended to investigate these barriers in depth, as they are potentially modifiable and can be addressed. Improving adherence to dietary recommendations has been shown to result in improved outcomes both within this study and others (Capuano et al, 2008; Hopanci Bicakli et al, 2017). The characteristics of this patient population also suggest that adherence may be particularly challenging given the high rate of mental health problems, substance use and social issues which increases psychologic distress and depression (DiMatteo et al, 2000; Kugaya et al, 2000). Adherence to other aspects of clinical management, such as adherence to radiotherapy protocols by the clinician, has also been shown to influence patient outcomes (Peters et al, 2010).

The main limitation of this study is that, despite a motivated patient cohort, poor adherence to the intervention impacted on the power of the study which may also explain why no significant differences were found on the post hoc per-protocol analysis. Steps were taken through the study by the research dietitian to improve adherence such as; increased communication to the multidisciplinary team and more regular contact with the intervention patients. The clinician staff did change over the course of the study period, which may have led to variations in clinical practice, however this is reflective of the pragmatic nature of nutrition care delivery in healthcare settings, and was minimised as much as possible, particularly for the intervention care arm. Whilst attrition from the study was generally very low, geographical location of patients did inhibit some follow-up care for the study, particularly for physical measurement of BIA. Our study identified P16 status as a strong confounding variable associated with a number of outcomes which was adjusted for in the multivariate models, however, given the impact of HPV status on survival outcomes
(Ang et al, 2010) it is increasingly being suggested that clinical trials should stratify for smoking, staging and HPV (P16) status. Differences in quality of life related to tube feeding may also not have been addressed with the tools used; however, they are the most widely accepted tools used in this population (Ojo et al, 2012). Future studies could consider additional tools specifically related to tube feeding, such as the QOL-EF (Stevens et al, 2011). Finally, swallowing outcomes were not measured using a validated assessment tool in this study, but there was no impact of long term gastrostomy dependency as a surrogate measure of dysphagia.

This interventional approach is not only restricted to hospitals that utilise prophylactic gastrostomy placement. Although designed as a tube feeding intervention, it could still be used by sites who prefer the reactive approach to nutrition management during treatment, as the supplements could be prescribed orally, and this is actually what some of our patients preferred to do. They were still included in the study as their nutritional intake was still being supplemented whether it was by the oral or enteral route. Only $11 \%$ of eligible patients (19 out of 174) declined to participate, which demonstrated a high acceptability of the trial, although this did not translate into adherence.

The use of prophylactic gastrostomy tubes remained high in this cohort of pre-defined high nutritional risk patients, with $87 \%$ of all study patients using their tube at the end of treatment, which confirms appropriate decision-making regarding tube placement. Although the early nutrition intervention did not minimise weight loss or improve other associated outcomes, this study has highlighted the significant barriers that patients encounter. Given the finding that patients who followed the early nutrition intervention had higher levels of adherence to nutrition recommendations later on in treatment, which did reduce weight loss, it is planned to introduce this approach into practice.

In conclusion, this research demonstrates the high complexity of managing patients with head and neck cancer, as desired outcomes have not been attained even with intensive intervention and support. While this novel early nutrition intervention can be considered to assist adherence, it is but one of many potential strategies. A multi-component intervention by the multidisciplinary team is recommended to adequately address and overcome patient barriers to healthcare recommendations before further improvements in nutrition outcomes are realised.

\section{ACKNOWLEDGEMENTS}

We thank the staff from the Combined Head and Neck Clinic for their support and access to patients for the study; the Department of Nutrition and Dietetics for the donation of enteral feeding supplies for the intervention; and David Smith, QIMR Berghofer Medical Research Institute for advice on statistical analysis. This research was supported by the following organisations awarding scholarships and funding for PhD study-Royal Brisbane and Women's Hospital Research Scholarships and Cancer Care Services (2012-2015) and the Sir Robert Menzies Memorial Scholarship in Allied Health Sciences (2016).

\section{CONFLICT OF INTEREST}

The authors declare no conflict of interest.

\section{REFERENCES}

Aaronson NK, Ahmedzai S, Bergman B, Bullinger M, Cull A, Duez NJ, Filiberti A, Flechtner H, Fleishman SB, de Haes JC et al. (1993) The European Organization for Research and Treatment of Cancer QLQ- 
C30: a quality-of-life instrument for use in international clinical trials in oncology. J Natl Cancer Inst 85: 365-376.

Ang KK, Harris J, Wheeler R, Weber R, Rosenthal DI, Nguyen-Tan PF, Westra WH, Chung CH, Jordan RC, Lu C, Kim H, Axelrod R, Silverman CC, Redmond KP, Gillison ML (2010) Human papillomavirus and survival of patients with oropharyngeal cancer. N Engl J Med 363: 24-35.

Bauer J, Capra S, Ferguson M (2002) Use of the scored patient-generated subjective global assessment (PG-SGA) as a nutrition assessment tool in patients with cancer. Eur J Clin Nutr 56: 779-785.

Beer KT, Krause KB, Zuercher T, Stanga Z (2005) Early percutaneous endoscopic gastrostomy insertion maintains nutritional state in patients with aerodigestive tract cancer. Nutr Cancer 52: 29-34.

Brown T, Banks M, Hughes B, Kenny L, Lin C, Bauer J (2014) Protocol for a randomized controlled trial of early prophylactic feeding via gastrostomy versus standard care in high risk patients with head and neck cancer. $B M C$ Nurs 13: 17

Brown T, Banks M, Hughes BG, Lin C, Kenny LM, Bauer JD (2015) New radiotherapy techniques do not reduce the need for nutrition intervention in patients with head and neck cancer. Eur J Clin Nutr 69: 1119-1124.

Brown TE, Getliffe V, Banks MD, Hughes BGM, Lin CY, Kenny LM, Bauer JD (2016) Validation of an updated evidence-based protocol for proactive gastrostomy tube insertion in patients with head and neck cancer. Eur $J$ Clin Nutr 70: 574-581.

Brown TE, Crombie J, Spurgin AL, Tripcony L, Keller J, Hughes BG, Dickie G, Kenny LM, Hodge RA (2016) Improving guideline sensitivity and specificity for the identification of proactive gastrostomy placement in patients with head and neck cancer. Head Neck 38(Suppl 1): E1163E1171.

Capuano G, Grosso A, Gentile PC, Battista M, Bianciardi F, Di Palma A, Pavese I, Satta F, Tosti M, Palladino A, Coiro G, Di Palma M (2008) Influence of weight loss on outcomes in patients with head and neck cancer undergoing concomitant chemoradiotherapy. Head Neck 30: 503-508.

Chang JH, Gosling T, Larsen J, Powell S, Scanlon R, Chander S (2009) Prophylactic gastrostomy tubes for patients receiving radical radiotherapy for head and neck cancers: a retrospective review. J Med Imaging Radiat Oncol 53: 494-499.

Clavel S, Fortin B, Despres P, Donath D, Soulieres D, Khaouam N, Charpentier D, Belair M, Guertin L, Nguyen-Tan PF (2011) Enteral feeding during chemoradiotherapy for advanced head-and-neck cancer: a single-institution experience using a reactive approach. Int J Radiat Oncol Biol Phys 79: 763-769.

Cocks K, King MT, Velikova G, de Castro Jr G, Martyn St-James M, Fayers PM, Brown JM (2012) Evidence-based guidelines for interpreting change scores for the European Organisation for the Research and Treatment of Cancer Quality of Life Questionnaire Core 30. Eur J Cancer 48: $1713-1721$.

Datema FR, Ferrier MB, Baatenburg de Jong RJ (2011) Impact of severe malnutrition on short-term mortality and overall survival in head and neck cancer. Oral Oncol 47: 910-914.

De Carvalho TM, Miguel Marin D, Da Silva CA, De Souza AL, Talamoni M, Lima CS, Monte Alegre S (2015) Evaluation of patients with head and neck cancer performing standard treatment in relation to body composition, resting metabolic rate, and inflammatory cytokines. Head Neck 37: 97-102.

DiMatteo MR, Lepper HS, Croghan TW (2000) Depression is a risk factor for noncompliance with medical treatment: meta-analysis of the effects of anxiety and depression on patient adherence. Arch Intern Med 160: 2101-2107.

Fayers PM, Aaronson NK, Bjordal K, Groenvold M, Curran D, Bottomley, on behalf of the EORTC Quality of Life Group (eds). The EORTC QLQ-C30 Scoring Manual, 3rd edn. European Organisation for Research and Treatment of Cancer: Brussels, Belgium, 2001.

Garg S, Yoo J, Winquist E (2010) Nutritional support for head and neck cancer patients receiving radiotherapy: a systematic review. Support Care Cancer 18: 667-677.

Gorenc M, Kozjek NR, Strojan P (2015) Malnutrition and cachexia in patients with head and neck cancer treated with (chemo)radiotherapy. Rep Pract Oncol Radiother 20: 249-258.

Hebuterne X, Lemarie E, Michallet M, De Montreuil CB, Schneider SM, Goldwasser F (2014) Prevalence of malnutrition and current use of nutrition support in patients with cancer. JPEN J Parenter Enteral Nutr 38: 196-204.
Hocking JS, Stein A, Conway EL, Regan D, Grulich A, Law M, Brotherton JM (2011) Head and neck cancer in Australia between 1982 and 2005 show increasing incidence of potentially $\mathrm{HPV}$-associated oropharyngeal cancers. Br J Cancer 104: 886-891.

Hopanci Bicakli D, Ozkaya Akagunduz O, Meseri Dalak R, Esassolak M, Uslu R, Uyar M (2017) The effects of compliance with nutritional counselling on body composition parameters in head and neck cancer patients under radiotherapy. J Nutr Metab 2017: 8631945.

Hubbard GP, Elia M, Holdoway A, Stratton RJ (2012) A systematic review of compliance to oral nutritional supplements. Clin Nutr 31: 293-312.

Isenring E, Capra S, Bauer J, Davies PS (2003) The impact of nutrition support on body composition in cancer outpatients receiving radiotherapy. Acta Diabetol 40(Suppl 1): S162-S164.

Isenring E, Zabel R, Bannister M, Brown T, Findlay M, Kiss N, Loeliger J, Johnstone C, Camilleri B, Davidson W, Hill J, Bauer J (2013) Updated evidence-based practice guidelines for the nutritional management of patients receiving radiation therapy and/or chemotherapy. Nutr Diet 70: 312-324.

Isenring EA, Capra S, Bauer JD (2004) Nutrition intervention is beneficial in oncology outpatients receiving radiotherapy to the gastrointestinal or head and neck area. Br J Cancer 91: 447-452.

Jager-Wittenaar H, Dijkstra PU, Vissink A, Langendijk JA, van der Laan BF, Pruim J, Roodenburg JL (2011) Changes in nutritional status and dietary intake during and after head and neck cancer treatment. Head Neck 33: 863-870.

Kiss N, Isenring E, Gough K, Wheeler G, Wirth A, Campbell BA, Krishnasamy M (2016) Early and intensive dietary counseling in lung cancer patients receiving (chemo)radiotherapy-a pilot randomized controlled trial. Nutr Cancer 68: 958-967.

Kugaya A, Akechi T, Okuyama T, Nakano T, Mikami I, Okamura H, Uchitomi Y (2000) Prevalence, predictive factors, and screening for psychologic distress in patients with newly diagnosed head and neck cancer. Cancer 88: 2817-2823.

Langius JA, Bakker S, Rietveld DH, Kruizenga HM, Langendijk JA, Weijs PJ, Leemans CR (2013a) Critical weight loss is a major prognostic indicator for disease-specific survival in patients with head and neck cancer receiving radiotherapy. Br J Cancer 109: 1093-1099.

Langius JA, Zandbergen MC, Eerenstein SE, van Tulder MW, Leemans CR, Kramer MH, Weijs PJ (2013b) Effect of nutritional interventions on nutritional status, quality of life and mortality in patients with head and neck cancer receiving (chemo)radiotherapy: a systematic review. Clin Nutr 32: 671-678.

Lee H, Havrila C, Bravo V, Shantz K, Diaz K, Larner J, Read P (2008) Effect of oral nutritional supplementation on weight loss and percutaneous endoscopic gastrostomy tube rates in patients treated with radiotherapy for oropharyngeal carcinoma. Support Care Cancer 16: 285-289.

Marcy PY, Magne N, Bensadoun RJ, Bleuse A, Falewee MN, Viot M, Bruneton JN (2000) Systematic percutaneous fluoroscopic gastrostomy for concomitant radiochemotherapy of advanced head and neck cancer: optimization of therapy. Support Care Cancer 8: 410-413.

Martin L, Senesse P, Gioulbasanis I, Antoun S, Bozzetti F, Deans C, Strasser F, Thoresen L, Jagoe RT, Chasen M, Lundholm K, Bosaeus I, Fearon KH, Baracos VE (2015) Diagnostic criteria for the classification of cancerassociated weight loss. J Clin Oncol 33: 90-99.

Merrick S, Farrell D (2012) Head and neck cancer patients' experiences of percutaneous endoscopic gastrostomy feeding: a Q-methodology study. Eur J Cancer Care 21: 493-504.

Moroney LB, Helios J, Ward EC, Crombie J, Wockner LF, Burns CL, Spurgin AL, Blake C, Kenny L, Hughes BG (2017) Patterns of dysphagia and acute toxicities in patients with head and neck cancer undergoing helical IMRT + / - concurrent chemotherapy. Oral Oncol 64: 1-8.

Nguyen NP, Norht D, Smith HJ, Dutta S, Alfieri A, Karlsson U, Lee H, Martinez T, Lemanski C, Nguyen LM, Ludin A, Sallah S (2006) Safety and effectiveness of prophylactic gastrostomy tubes for head and neck cancer patients undergoing chemoradiation. Surg Oncol 15: 199-203.

Nugent B, Lewis S, O’Sullivan JM (2013) Enteral feeding methods for nutritional management in patients with head and neck cancers being treated with radiotherapy and/or chemotherapy. Cochrane Database Syst Rev 1: CD007904.

Nugent B, Parker MJ, McIntyre IA (2010) Nasogastric tube feeding and percutaneous endoscopic gastrostomy tube feeding in patients with head and neck cancer. J Hum Nutr Diet 23: 277-284. 
Ojo B, Genden EM, Teng MS, Milbury K, Misiukiewicz KJ, Badr H (2012) A systematic review of head and neck cancer quality of life assessment instruments. Oral Oncol 48: 923-937.

Olson R, Karam I, Wilson G, Bowman A, Lee C, Wong F (2013) Populationbased comparison of two feeding tube approaches for head and neck cancer patients receiving concurrent systemic-radiation therapy: is a prophylactic feeding tube approach harmful or helpful? Support Care Cancer 21: 3433-3439.

Orphanidou C, Biggs K, Johnston ME, Wright JR, Bowman A, Hotte SJ, Esau A, Myers C, Blunt V, Lafleur M, Sheehan B, Griffin MA (2011) Prophylactic feeding tubes for patients with locally advanced head-andneck cancer undergoing combined chemotherapy and radiotherapysystematic review and recommendations for clinical practice. Curr Oncol 18: e191-e201.

Paccagnella A, Morello M, Da Mosto MC, Baruffi C, Marcon ML, Gava A, Baggio V, Lamon S, Babare R, Rosti G, Giometto M, Boscolo-Rizzo P, Kiwanuka E, Tessarin M, Caregaro L, Marchiori C (2010) Early nutritional intervention improves treatment tolerance and outcomes in head and neck cancer patients undergoing concurrent chemoradiotherapy. Support Care Cancer 18: 837-845.

Peters LJ, O'Sullivan B, Giralt J, Fitzgerald TJ, Trotti A, Bernier J, Bourhis J, Yuen K, Fisher R, Rischin D (2010) Critical impact of radiotherapy protocol compliance and quality in the treatment of advanced head and neck cancer: results from TROG 02.02. J Clin Oncol 28: 2996-3001.

Rabinovitch R, Berkey B, Raben D, Cooper J (2007) Reply to letter to the editor. Head Neck 29: 519-520.

Rabinovitch R, Grant B, Berkey BA, Raben D, Aang KK, Fu KK, Cooper JS. for the Radiation Therapy Oncology Group (2006) Impact of nutrition support on treatment outcome in patients with locally advanced head and neck squamous cell cancer treated with definitive radiotherapy: a secondary analysis of RTOG trial 90-03. Head Neck 28: 287-296.

Ravasco P, Monteiro-Grillo I, Vidal PM, Camilo ME (2004) Cancer: disease and nutrition are key determinants of patients' quality of life. Support Care Cancer 12: 246-252.

Rayker A, Correa L, Russo L, Brown P, Lee N, Pfister D, Gerdes H, Shah J, Kraus D, Schattner M, Shike M (2009) The role of pretreatment percutaneous endoscopic gastrostomy in facilitating therapy of head and neck cancer and optimizing the body mass index of the obese patient. JPEN J Parenter Enteral Nutr 33: 404-410.

Rutter CE, Yovino S, Taylor R, Wolf J, Cullen KJ, Ord R, Athas M, Zimrin A, Strome S, Suntharalingam M (2011) Impact of early percutaneous endoscopic gastrostomy tube placement on nutritional status and hospitalization in patients with head and neck cancer receiving definitive chemoradiation therapy. Head Neck 33: 1441-1447.
Salas S, Baumstarck-Barrau K, Alfonsi M, Digue L, Bagarry D, Feham N, Bensadoun RJ, Pignon T, Loundon A, Deville JL, Zanaret M, Favre R, Duffaud F, Auquier P (2009) Impact of the prophylactic gastrostomy for unresectable squamous cell head and neck carcinomas treated with radiochemotherapy on quality of life: Prospective randomized trial. Radiother Oncol 93: 503-509.

Scolapio JS, Spangler PR, Romano MM, McLaughlin MP, Salassa JR (2001) Prophylactic placement of gastrostomy feeding tubes before radiotherapy in patients with head and neck cancer: is it worthwhile? J Clin Gastroenterol 33: 215-217.

Silander E, Nyman J, Bove M, Johansson L, Larsson S, Hammerlid E (2012) Impact of prophylactic percutaneous endoscopic gastrostomy on malnutrition and quality of life in patients with head and neck cancer: a randomized study. Head Neck 34: 1-9.

Splett PL (1996) Cost Outcomes of Nutrition Intervention. Part 2. Mead Johnson and Company: New York, NY, USA.

Stevens CS, Lemon B, Lockwood GA, Waldron JN, Bezjak A, Ringash J (2011) The development and validation of a quality-of-life questionnaire for head and neck cancer patients with enteral feeding tubes: the QOL-EF. Support Care Cancer 19: 1175-1182.

Talwar B, Findlay M (2012) When is the optimal time for placing a gastrostomy in patients undergoing treatment for head and neck cancer? Curr Opin Support Palliat Care 6: 41-53.

Van den Broek PW, Rasmussen-Conrad EL, Naber AH, Wanten GJ (2009) What you think is not what they get: significant discrepancies between prescribed and administered doses of tube feeding. Br J Nutr 101: 68-71.

Wang J, Liu M, Liu C, Ye Y, Huang G (2014) Percutaneous endoscopic gastrostomy versus nasogastric tube feeding for patients with head and neck cancer: a systematic review. J Radiat Res 55: 559-567.

Wiggenraad RG, Flierman L, Goossens A., Brand R, Verschuur HP, Croll GA, Moser LE, Vriesendorp R (2007) Prophylactic gastrostomy placement and early tube feeding may limit loss of weight during chemoradiotherapy for advanced head and neck cancer, a preliminary study. Clin Otolaryngol 32: 384-390.

Zhang Z, Zhu Y, Ling Y, Zhang L, Wan H (2016) Comparative effects of different enteral feeding methods in head and neck cancer patients receiving radiotherapy or chemoradiotherapy: a network meta-analysis. Onco Targets Ther 9: 2897-2909.

This work is published under the standard license to publish agreement. After 12 months the work will become freely available and the license terms will switch to a Creative Commons AttributionNonCommercial-Share Alike 4.0 Unported License.

Supplementary Information accompanies this paper on British Journal of Cancer website (http://www.nature.com/bjc) 